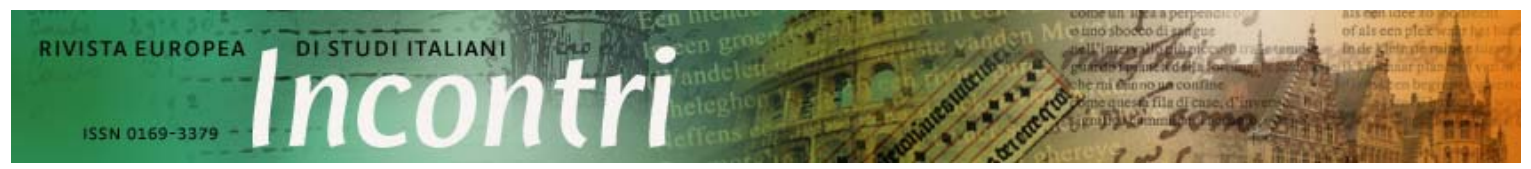

URN:NBN:NL:Ul:10-1-114247 - Publisher: Igitur publishing

Content is licensed under a Creative Commons Attribution 3.0 License

Anno 28, 2013 / Fascicolo 1 - Website: www.rivista-incontri.nl

\title{
Riscrivere la storia Modalità di rappresentazione del colonialismo italiano in Albania
}

\author{
Daniele Comberiati
}

\section{Gli 'italianesi': portatori di un'identità ibrida}

Mia madre mi parlava sempre di mio padre e mi raccontava dell'Italia, e io ho cominciato a venirci ogni notte in Italia, nei miei sogni. Ho iniziato a sentire il rumore del mare, a immaginare come potesse essere Ischia, le montagne. Conoscevo l'Italia senza averla mai vista.

Al punto che quando poi siamo tornati veramente in Italia [...], scendendo a Roma dal treno io m'aspettavo un'orchestra, con la gente che suonava ballava e cantava. E invece non suonava e ballava nessuno, e tantomeno cantava nessuno. Anzi, ci hanno tenuti bloccati cinque giorni in questura e zitti, e se reclamavamo ci guardavano pure storto e zitti lo stesso. "Ma guarda st'albanesi...", dicevano i poliziotti.

Le citazioni provengono da un testo teatrale del 2011 ad opera del drammaturgo calabrese Saverio La Ruina, dal titolo particolarmente riuscito Italianesi, ${ }^{1}$ una contrazione fra 'italiani' e 'albanesi'. La storia, a dire il vero poco conosciuta, è sintomatica delle conflittualità postcoloniali insorte fra Italia e Albania: nel 1951, alcuni tecnici italiani rimasti in Albania per lavorare alla ricostruzione del paese dopo la Seconda guerra mondiale, vennero improvvisamente rimpatriati e considerati 'persone non gradite' dal governo albanese, a causa di non meglio specificate operazioni sovversive. In Albania rimasero però le loro mogli (in alcuni casi italiane, in altre autoctone) e i loro figli, che vennero internati in un centro per stranieri potenzialmente pericolosi. I giovani crebbero così senza 'padre' e 'patria', considerando il loro legame con la figura paterna e con l'Italia il fulcro di un'identità costruita per difetto, attraverso continue assenze e mancanze. Il rapporto con l'Italia venne ovviamente interrotto e anche la corrispondenza era vietata o nel migliore dei casi censurata. Spesso i tecnici italiani rimpatriati si erano rifatti una vita e non avevano più contatti con mogli e figli lasciati in Albania. A ben vedere, il luogo in cui questi ragazzi crescono rappresenta un elemento focale della condizione coloniale e postcoloniale; la 'forma campo', ${ }^{2}$ infatti, si costituisce come un luogo asfittico, dove la sospensione dei diritti civili si accompagna, in un tipico connubio coloniale, ad una limitazione del corpo. $\grave{E}$ l'esempio di segregazione portato all'estremo, laddove la divisione tra colonizzatori e

\footnotetext{
${ }^{1}$ Italianesi, di S. La Ruina, con S. La Ruina, 2011.

${ }^{2}$ Cfr. F. Rahola, 'La forma campo. Per una genealogia dei luoghi di transito e di internamento del presente', in: Conflitti globali II, 4 (2006), pp. 11-27.
} 
colonizzati o tra autoctoni e stranieri non appare dissimile da tanti altri esempi che possono essere citati nel passato ma anche nel presente, dalla divisione fra quartieri di bianchi occidentali e di neri nella Addis Abeba coloniale, fino, sempre in ambito italiano, ai contemporanei Cpt (Centri di Permanenza Temporanea), che ai centri per stranieri in Albania assomigliano terribilmente. Nel 1991, dopo la caduta del regime di Hoxha e grazie alla cosiddetta 'operazione Cora', trecentosessantacinque figli di italiani, dunque 'italianesi', giungono in Italia, in quella che teoricamente avevano sempre considerato la loro patria, pur non conoscendola e in rari casi parlandone la lingua, visto che di fatto erano nati e cresciuti in Albania. Ecco che la diffidenza e il razzismo provato in Italia modellano ancora la loro identità per difetto: se prima erano 'gli italiani', figli di nemici del popolo, ora diventano 'gli albanesi', avanguardia elitaria di una nuova ondata migratoria che si sarebbe presto riversata sulle coste italiane.

La loro storia, con la costruzione di una patria lontana e irreale dapprima necessaria per sopravvivere ma che in seguito li rifiuta, a ben vedere è, per sommi capi, vicina alle narrazioni delle migrazioni di 'ritorno', soprattutto a quelle inerenti alla storia e alla politica coloniale. Ricordano, questi 'italianesi', i ben più numerosi italiani che nel 1970 nelle navi bianche giungono in Italia dalla Libia. ${ }^{3}$ Anche in quel caso, tra l'altro, il loro approdo fu delimitato da un campo, nel tentativo irrealizzabile di confinare la memoria e il passato coloniali. Non dissimili appaiono le storie private degli 'insabbiati' o degli 'incatramati', ${ }^{4}$ italiani rimasti in Etiopia dopo la fine delle colonie: la maggior parte di loro, dopo aver tentato di tornare in Italia ed essersi sentita 'rifiutata' o 'straniera in patria', decise di rimanere nell'entroterra di quella terra che avrebbe dovuto costituire il nuovo impero, trovando lavoro nel trasporto di materiali con $i$ camion o aprendo locande in luoghi sperduti. In ambito non italiano, non va dimenticato ad esempio che il contesto della guerra d'Algeria si profilò tanto drammatico, anche per la compresenza dei pieds-noirs nel territorio algerino e di una cospicua comunità algerina in Francia, elemento che contribui a rimettere in discussione il connubio popolo/nazione. Non troppo diversa dall'accoglienza riservata dall'Italia nei confronti degli 'italianesi', fu quella con cui il Portogallo accolse i retornados dalla guerra d'Angola: ${ }^{5}$ portatori di una sconfitta militare e soprattutto della fine del sogno coloniale del grande Portogallo, $i$ retornados vennero schedati e collocati temporaneamenti in vecchi hotel alla periferia di Lisbona, in attesa di essere reinseriti nel contesto portoghese. Quelle citate sono tutte figure ibride, che portano sulla propria storia, e spesso sul proprio corpo, i segni delle conflittualità e delle contraddizioni della dinamica coloniale.

\section{Il confine liquido del mar Adriatico}

Tornando al contesto albanese, le tensioni con l'Italia da cui in seguito è scaturita la vicenda degli 'italianesi', derivano, è bene ricordarlo, dall'occupazione da parte del regime fascista del 1939. Tale occupazione è solo una parte, per quanto decisiva, di una storia intrecciata che, durante gli anni della Guerra fredda, sembra essere dimenticata. Alla caduta delle frontiere che dividono i due blocchi, un evento 'portante' sembra voler far riaffiorare la memoria. Nell'agosto del 1991, al porto di Bari, da una vetusta nave da carico, la Vlora, iniziarono a scendere centinaia di albanesi. Dopo essere stati ammassati

\footnotetext{
${ }^{3}$ Cfr. A.L. Pachera, Tripoli 1970: allontanati dalla nostra vita, Rovereto (Tn), Osiride, 2010.

${ }^{4} \mathrm{~T}$. Besozzi, Il sogno del settimo viaggio, Roma, Fazi, 2001.

${ }^{5}$ Cfr. il romanzo di Antonio Lobo Antunes, As Naus, Lisboa, D. Qioxote, 1988; trad. it. Le navi, Torino, Einaudi, 1997.
} 
dalle autorità di polizia nello stadio della città, la maggior parte di loro venne quasi immediatamente rimpatriata, non prima però che le condizioni nello stadio divenissero disagevoli. ${ }^{6}$ In un ampio resoconto pubblicato il 12 agosto sul Corriere della Sera, Enzo Biagi parlò del 'sogno svanito' degli albanesi, mettendolo in relazione con il 'sogno svanito' degli italiani: 'È svanito il sogno degli albanesi, ma anche quello degli italiani. La quinta potenza industriale del mondo non è in grado, in tre giorni, di distribuire diecimila tazze di caffelatte'. ${ }^{7}$

Le parole di Biagi possono far pensare, attraverso la delusione condivisa, ad uno spaccato di storia comune che, impietosamente, viene ricordato dallo sbarco massiccio dalla Vlora; ${ }^{8}$ la prossimità della distanza - due coste che sembrano toccarsi, o comunque si specchiano - è amplificata dallo iato culturale e politico perpetuatosi dal dopoguerra in poi. Il socialismo reale albanese con la dittatura di Hoxha, l'entrata dell'Italia nella Nato e gli aiuti del piano Marshall sembravano aver centuplicato la striscia di mare che divide i due paesi. Ė però un semplice atto fisico, l'attraversamento dell'Adriatico, a far crollare una frontiera inconsistente e a causare un cortocircuito storico che rimette in discussione le relazioni fra Italia e Albania. Quello stesso mare, infatti, era stato attraversato in senso contrario alla fine degli anni Trenta, in occasione dell'invasione italiana. Sempre sul Corriere della Sera, veniva pubblicato l'ultimatum spedito al re Zog:

Amici albanesi!

Le truppe italiane che sbarcano oggi sulla vostra terra sono le truppe di un popolo che vi è stato amico nei secoli e ve lo ha dimostrato.

Non opponete vane resistenze che sarebbero stroncate. Non ascoltate gli uomini del Governo che vi hanno immiseriti e che vorrebbero ora condurvi ad un inutile spargimento di sangue.

Le truppe di Sua Maestà il Re d'Italia e Imperatore vengono e rimarranno il tempo necessario per ristabilire l'ordine, la giustizia, la pace. ${ }^{9}$

Il riferimento a 'un popolo che vi è stato amico nei secoli' è particolarmente indicativo: è ancora il confine liquido del mar Adriatico a portarci indietro nel tempo, precisamente al quindicesimo secolo, quando gli albanesi iniziarono una strenua resistenza contro $i$ turchi. A tale periodo viene fatta risalire la presenza delle prime comunità albanesi in Italia, i cosiddetti arbëreshe, che guidati da Scanderbeg raggiunsero le coste dell'Italia per fuggire dall'invasione ottomana.

Se il mare porta i segni dei passaggi, delle guerre e delle invasioni, ${ }^{10}$ inglobati dall'acqua e più colpevolmente cancellati dalle memorie, la toponomastica delle città, linguaggio stavolta in superficie ma non per questo facilmente interpretabile, propone una mappatura della storia che parte dai nomi e dalla scrittura. In piazza Albania, a Roma, non lontano dalla basilica di San Pietro, una statua in bronzo di Scanderbeg è immobile a ricordare gli scambi fra i due paesi, e quel passing adriatico che, almeno in

\footnotetext{
${ }^{6}$ Cfr. a tale proposito P. Ginsborg, L'Italia del tempo presente. Famiglia, società civile, stato, 1980-1996, Torino, Einaudi, 1998, pp. 121-128.

${ }^{7}$ E. Biagi, Niente spaghetti, in: Corriere della Sera (12 agosto 1991), p. 19.

${ }^{8}$ Sul medesimo evento cfr. il recente documentario di D. Vicari, La nave dolce, Italia, 2012, nel quale vengono intervistati alcuni membri dell'equipaggio e sono ripercorse le reazioni politiche e mediatiche italiane.

${ }^{9} \mathrm{Cfr}$. 'Amici albanesi, non opponete vane resistenze', in: Corriere della Sera (8 aprile 1939), p. 1.

${ }^{10} \mathrm{Cfr}$. sul tema I. Chambers, 'Maritime Criticism and Lessons from the Sea', in: Insights, 9, 3 (2010), consultabile sul sito http://www.dur.ac.uk/resources/ias/insights/Chambers30Mar.pdf (22 febbraio 2013).
} 
tre decisivi momenti storici (l'arrivo degli albanesi nel millecinquecinquecento, l'invasione fascista e gli sbarchi degli anni Novanta) ha mutato profondamente anche la conformazione interna dei due stati. Ai piedi della statua (eretta nel 1940, un anno dopo l'invasione, dallo scultore Romanelli), una lapide apposta nel 1968, in occasione del cinquecentenario dell'arrivo degli arbëreshe, ricorda l'eroe albanese come 'difensore della civiltà occidentale', schiacciando l'Albania verso l'Italia e eliminando, in chiave anti-turca e anti-islamica, le differenze.

Se alla grande Storia ufficiale affianchiamo storie meno note, eppure decisive per comprenderne le ragioni e le sfumature, innumerevoli sono le tracce di un percorso intrecciato. Ad uno sguardo attento non può sfuggire che anche l'invasione dell'Albania nel 1939 riporta ad una serie di fatti e di eventi che, nel loro insieme, sono da considerare coloniali e postcoloniali. Lo sguardo dell'Italia verso oriente ha dei precisi riferimenti storici: quell'area complessa che dalla Slovenia ai Balcani giunge fino alla Grecia ha storicamente attirato le mire espansionistiche italiane. Al di là delle isole del Dodecaneso, conquistate insieme alla Libia dopo la guerra italo-turca, le questioni a lungo irrisolte di Fiume, di Trieste e dell'Istria mostrano come l'ampliamento a est dei propri confini nazionali sia stato un tema molto dibattutto già negli anni immediatamente precedenti l'unità. Come nel caso dell'acquisizione del porto di Assab, avvenuto prima che Roma venisse sottratta allo Stato Pontificio, contribuendo in tal modo a costruire un'identità nazionale collegata fin dai suoi albori all'espansione coloniale, così il dibattito sui confini orientali della nazione si è sviluppato negli anni dell'indipendenza, facendo intravedere immediatamente come si sarebbero prospettati gli scenari futuri. Se tali aspettative 'orientali' erano da considerarsi come un tentativo di sopravanzare l'influenza ottomana nell'area, dal secondo dopoguerra in poi, in particolar modo negli anni della Guerra Fredda, la condizione dell'Italia di frontiera/cerniera orientale ha assunto un peso specifico all'interno della dinamica di contrapposizione dei due blocchi. È possibile pensare al mar Adriatico in quegli anni come a un lungo confine che parte dalla frontiera del Friuli fino a giungere alle coste pugliesi che guardano l'Albania.

Il fatto che l'occupazione vera e propria dell'Albania non sia durata che pochi anni, non cancella la tensione storica verso i suoi confini, né l'influenza che la cultura popolare italiana (esemplificata soprattutto dai programmi della televisione nazionale) ha esercitato sulla popolazione albanese dagli anni Sessanta in poi. In quest'ottica è di grande aiuto un saggio di Luca Scarlini, in cui è proposto un ampliamento del concetto di 'letteratura d'oltremare', che a suo dire va esteso anche a paesi che non furono direttamente colonizzati dall'Italia, come ad esempio la Tunisia, ma verso i quali le mire espansionistiche e le pressioni politiche e culturali italiane furono sempre costanti. ${ }^{11}$ L'Italia, in effetti, conquistò alcuni paesi senza un progetto politico preciso, ma solo perché in quel momento erano più deboli e quindi più facilmente attaccabili o perché li considerava un ripiego rispetto ad altre mete più ambite (è il caso della Libia, che fu frettolosamente occupata perché la Tunisia, vero obiettivo di Crispi, era già entrata nell'orbita francese).

È il concetto stesso di 'influenza coloniale' italiana che va ripensato: è fondamentale in tal senso riaffermare come postcoloniali le narrazioni provenienti dall'Albania. Il rapporto fra Italia e Albania ha in questi testi un'importanza

\footnotetext{
${ }^{11}$ L. Scarlini, 'Posti al sole e giochi di specchi: per una storia della letteratura italiana d'Oltremare', in: Nuovi Argomenti LVI, 43 (2008), pp. 56-75.
} 
predominante: si potrebbe anzi asserire che quella italiana nei confronti del paese balcanico sia stata una doppia colonizzazione, nata in principio con l'invasione del 1939 e proseguita attraverso l'influenza della televisione italiana sull'immaginario collettivo albanese durante gli ultimi anni del comunismo, elemento che ha comportato l'identificazione dell'Italia come 'terra promessa' durante gli sbarchi dei primi anni Novanta. In tale prospettiva, anche le considerazioni di scrittrici come Ornela Vorpsi, Anilda Ibrahimi e Elvira Dones o di autori come Ron Kubati e Leonard Guaci, sulla definizione di Italia come 'terra promessa' acquistano una valenza più complessa. Se il mito occidentale si dimostra effimero dopo l'emigrazione, esso va ripensato, dal punto di vista postcoloniale, rispetto agli effetti che ha avuto sull'immaginario collettivo, tenendo presente come $\mathrm{i}$ processi culturali influiscano sulle evoluzioni socioeconomiche. D'altra parte anche Laura Ricci, nella sua ricca analisi sul linguaggio coloniale italiano, ${ }^{12}$ si era interrogata sulla valenza e sulle tipologie di significato dell'espressione 'colonialismo', giungendo infine alla conclusione che il colonialismo travalica l'espansione coloniale, poiché in realtà riguarda da una parte la riorganizzazione della colonia sulla falsa riga della metropoli e dall'altra le reazioni che la 'colonia' - intesa in senso lato come area di influenza - mostra rispetto alle sollecitazioni culturali della metropoli.

\section{Gli scrittori migranti provenienti dall' Albania}

Veniamo in maniera più precisa agli autori presi in considerazione. All'interno della produzione letteraria di scrittori albanesi in lingua italiana si possono distinguere almeno due generazioni: la prima, esemplificata dall'opera del poeta Gëzim Hajdari, ${ }^{13}$ rappresenta autori che hanno passato gran parte della loro esistenza sotto il regime di Hoxha e che solo negli anni Novanta hanno avuto la possibilità di scrivere senza essere censurati. La seconda invece è costituita da un 'macro-insieme', che si estende dal trittico di scrittrici Elvira Dones-Anilda Ibrahimi-Ornela Vorpsi, fino alle opere di Leonard Guaci e Ron Kubati. Sono tutti scrittori che, pur presentando storie personali completamente diverse, hanno iniziato a scrivere 'dopo' la dittatura di Hoxha e grosso modo (a parte la Dones) appartengono alla generazione nata fra la fine degli anni Sessanta e la prima metà dei Settanta, che ha vissuto sotto il comunismo l'infanzia e l'adolescenza, ma ha avuto la possibilità di emigrare in giovane età e di pubblicare le prime opere senza censura.

Le differenze generazionali sono esemplificate dal caso di Hajdari, il primo autore migrante a vincere un premio letterario ufficiale, poiché nel $1997 \mathrm{gli}$ fu assegnato il Premio Montale per la poesia inedita. Il regime di Hoxha ha certamente influito sulla sua evoluzione letteraria, e d'altronde è inevitabile che, per la sua storia personale, il riferimento a un passato dittatoriale assuma una valenza specifica. 'lo parto verso il vuoto / a gridare del sangue versato / su questo territorio sterile' canta Gëzim Hajdari, associando la funzione etica della poesia civile (documentare la dittatura, in questo caso) alla rabbia per essere stato costretto a lasciare il paese natale e alle difficoltà dell'approdo nel paese di accoglienza. Nella sua poesia quel 'vuoto' inizia poi a prendere forma: è l'Italia, paese del quale impiega la lingua letteraria e in cui ha scelto di vivere. È evidente come la provenienza da un paese sotto dittatura renda per lo meno

\footnotetext{
${ }^{12}$ L. Ricci, La lingua dell'impero. Comunicazione, letteratura e propaganda nell'età del colonialismo italiano, Roma, Carocci, 2005.

${ }^{13}$ G. Hajdari, Poesie scelte 1990-2007, Roma, Controluce, 2007; cfr. per quanto riguarda la critica A. Gazzoni, Poesia dell'esilio. Saggi su Gëzim Hajdari, Isernia, Cosmo lannone, 2010.
} 
inutili le speculazioni nostalgiche di certa letteratura migrante: se vi è un luogo ameno e ideale a cui la memoria può fare riferimento, tale luogo sarà sempre al di là del regime, in un tempo storico in cui il partito non aveva ancora preso il potere oppure, più frequentemente, in un tempo personale (l'infanzia, l'adolescenza o piuttosto un tempo mitico e irreale) in cui la portata della dittatura non era ancora stata compresa. In tal caso il passaggio da un paese all'altro rappresenta anche l'entrata nel mondo occidentale: il breve spazio di mare che divide i due stati è in realtà una frontiera ben più difficile da valicare, poiché di fatto coincide con l'antica divisione fra mondo comunista e mondo capitalista.

Per quanto riguarda la generazione successiva, un innegabile motivo di interesse è contenuto nella specificità di genere. Il corpo femminile scisso, disprezzato e infine rivitalizzato di Vergine giurata di Elvira Dones è un elemento essenziale anche per comprendere le dinamiche del viaggio migratorio: si può cambiare corpo (e genere) come si cambiano una lingua, una cultura e un paese? Nel passaggio da una nazione all'altra e da un modo di vivere all'altro è insita anche una violenza che si ripercuote, inarrestabile, sul corpo. I corpi nelle opere di queste scrittrici vengono celati e nascosti (come accade in parte a Rosso come una sposa di Anilda Ibrahimi),${ }^{14}$ oppure, allo stesso modo delle fotografie che Ornela Vorpsi pubblica sulle copertine e all'interno dei suoi testi, amplificando lo spazio della propria scrittura, tendono a evocare un'atmosfera che è possibile 'sentire' attraverso la lettura. Nella stessa Vorpsi diviene fondamentale non solo l'atto del guardare (l'azione del Sehir, termine di origine turca che significa letteralmente 'guardare gli altri', assume da questo punto di vista un ruolo dominante nella sua poetica), ma anche il sentirsi oggetto dello sguardo altrui: lo sguardo maschile che opprime, deforma, adula o disprezza rischia di schiacciare l'identità femminile nelle sponde opposte del mar Adriatico.

Siamo così in presenza di una conflittualità che continua dopo la migrazione, un tentativo di far emergere la propria personalità minacciata da due versioni differenti del potere coercitivo maschile: l'Italia e l'Albania, o in senso più generale l'occidente e $i$ Balcani, sono accomunate dallo sguardo maschile e diventano il terreno per una ugualmente necessaria liberazione. La scrittrice italo-somala Igiaba Scego si diceva interessata, alcuni anni fa, alle reazioni che mostrano i corpi quando la storia li investe: che cosa succede ai personaggi delle autrici, e in generale degli scrittori albanesi, quando viene attraversato il mar Adriatico? Come in Educazione siberiana di Nicolai Lilin, ${ }^{15}$ il corpo diviene la mappa di una storia e la carta su cui vengono incisi i segni di un triplo passaggio: di epoche, di nazioni attraverso il mare, e, nel caso degli scrittori, di lingua. Da tale prospettiva il legame fra autobiografia e finzione accoglie un elemento ulteriore: la memoria delle vicende narrate, laddove ci si avvicini ai generi della nonfiction o della faction, si prospetta ovviamente come memoria selettiva, legata inscindibilmente ai segni che la storia, personale e collettiva, ha lasciato sul corpo. Indicativa risulta inoltre l'evoluzione tematica delle tre autrici: entrambe nei romanzi più recenti (La mano che non mordi per Vorpsi, Piccola guerra perfetta per Dones, L'amore e gli stracci del tempo per Ibrahimi) ${ }^{16}$ spostano l'asse di riferimento dalla relazione biunivoca fra Italia e Albania, ad un legame più complesso dell'Albania con

\footnotetext{
${ }^{14}$ O. Vorpsi, II paese dove non si muore mai, Torino, Einaudi, 2005; E. Dones, Vergine giurata, Milano, Feltrinelli, 2007; A.Ibrahimi, Rosso come una sposa, Torino, Einaudi, 2008.

${ }^{15} \mathrm{~N}$. Lilin, Educazione siberiana, Torino, Einaudi, 2009.

${ }^{16}$ O. Vorpsi, La mano che non mordi, Torino, Einaudi, 2007; A. Ibrahimi, L'amore e gli stracci del tempo, Torino, Einaudi, 2010; E. Dones, Piccola guerra perfetta, prefazione di R. Saviano, Torino, Einaudi, 2011.
} 
l'occidente e con i Balcani. L'allargamento di campo ovviamente comporta l'inserimento della tematica del conflitto balcanico, secondo punti di vista molteplici, dalla guerra in Kossovo di Ibrahimi e Dones ai ricordi 'fisici' di una città smembrata nella Sarajevo di Vorpsi. Si nota la necessità di riposizionare geograficamente e storicamente l'appartenenza albanese evitando il semplicistico connubio oriente/occidente, ma ponendola al centro delle delicate questioni etniche e politiche dei Balcani negli ultimi anni. L'Italia in questo caso non rappresenta più la terra promessa, ma una nazione colpevole dell'indifferenza dell'Europa durante gli anni di guerra.

D'altra parte la fantomatica 'terra promessa' si rivela illusoria fin dal romanzo d'esordio di Ron Kubati, Va e non torna, ${ }^{17}$ nel quale vengono messe in luce le contraddizioni dell'accoglienza italiana, concepita esclusivamente come assimilazione oppure come collocazione del migrante all'interno di ruoli e percorsi stereotipati. Anche nel romanzo I grandi occhi del mare di Leonard Guaci, ${ }^{18}$ il rapporto fra Italia e Albania si presenta come conflittuale: da una parte il mar Adriatico diventa il simbolo della prossimità culturale tra i due paesi; dall'altra però risulta evidente lo sbilanciamento, frutto di una precisa volontà di egemonia culturale, delle due nazioni nel processo di scambio, laddove una 'comunità' (quella italiana) produce immagini e l'altra le riceve e le assorbe. La televisione è il medium di questa dialettica, nel caso specifico la trasmissione dei programmi italiani (visibili clandestinamente in Albania) dal 1960 al 1990. Nei due autori il discorso di genere è declinato secondo parametri differenti: nel loro caso, soprattutto per quanto riguarda Kubati, la relazione con la donna italiana o occidentale diventa il mezzo per sentirsi realmente integrati nel paese di accoglienza e la relazione sentimentale una metafora del controverso rapporto con l'Italia. In Va e non torna, ad esempio, il fallimento amoroso racchiude una serie di riflessioni più generali sulle difficoltà di integrazione e sull'incapacità del protagonista di radicarsi profondamente - dal punto di vista degli affetti e non solo da quello professionale - in un paese straniero.

\section{Per una conclusione}

L'approdo sulle coste italiane rappresenta, nelle contronarrazioni degli scrittori albanesi, un momento topico: gli 'italianesi' di La Ruina, il giovane protagonista di Guaci o la ragazza che giunge in Italia insieme alla madre in II paese dove non si muore mai di Vorpsi, vengono immediatamente 'respinti' dal punto di vista culturale. La diffidenza e l'ostilità si rivelano tanto grandi quanto erano state le aspettative. Attraversare il mare e fuggire da una dittatura non si dimostra sufficiente per sentirsi davvero liberi.

Tale conflittualità era già stata intuita, senza ritornare a esempi noti come il film Lamerica di Gianni Amelio, ${ }^{19}$ da Ismail Kadaré, che con il suo romanzo II generale dell'armata morta ${ }^{20}$ aveva creato un'efficace contronarrazione coloniale, mostrando come la guerra di occupazione del 1939 venisse considerata, da parte albanese, uno spartiacque fondamentale per quanto riguarda la percezione dell'Italia e le sue influenze culturali. All'interno di un percorso della memoria irregolare - per il quale non si può parlare propriamente di rimozione, quanto piuttosto di una memoria sommersa

\footnotetext{
${ }^{17}$ R. Kubati, Va e non torna, Nardò (Lecce), Besa, 1999.

${ }^{18}$ L. Guaci, I grandi occhi del mare, Nardò (Lecce), Besa, 2006.

19 Lamerica, regia di G. Amelio, con E. Lo Verso e M. Placido, produzione Mario \& Vittorio Cecchi Gori, Italia, 1994.

${ }^{20}$ I. Kadaré, Gjenerali i ushtrisë së vdekur, Tirana, Onufri, 1963; trad. it. Il generale dell'armata morta, Milano, Longanesi, 1982.
} 
che riemerge sporadicamente - gli eventi legati alla relazione con l'Albania subiscono un primo processo di ostruzione - nel senso che l'Italia attuale accetta con difficoltà di rivangarli e di rifletterci - e un secondo di traslazione, poiché, proprio attraverso le produzioni culturali analizzate, il discorso dal piano prettamento storico o politico si sposta su quello della rappresentazione e della rinegoziazione dell'identità nazionale.

Quella fra i due paesi, in conclusione, è una storia paradigmatica per cercare di analizzare le contraddizioni e le conflittualità derivate dal colonialismo. Vi sono pagine di storia rimossa che puntualmente riaffiorano (e in questo caso la metafora della nave da carico Vlora mi sembra particolarmente efficace), episodi celati o dimenticati (come le vicende degli 'italianesi'), tentativi di contronarrazione da parte degli scrittori migranti e postcoloniali attuali, che cercano di riannodare le fila di un passato non sempre semplice da interpretare. Queste scritture postcoloniali portano inevitabilmente ad un ripensamento della storia coloniale italiana ed albanese e mettono in discussione $i$ concetti di 'patria', 'nazione' e, soprattutto, di 'letteratura nazionale'.

\section{Parole chiave}

Postcolonialismo, Albania, letteratura della migrazione, colonialismo italiano, scrittrici migranti

Daniele Comberiati è chargé de Recherches Frs-Fnrs all'Université Libre de Bruxelles, presso cui ha conseguito il dottorato di ricerca nel 2008. Ha pubblicato la raccolta di interviste La quarta sponda. Scrittrici in viaggio dall'Africa coloniale all'Italia di oggi (Roma, Caravan, 2009), i saggi Scrivere nella lingua dell'altro. La letteratura degli immigrati in Italia (1989-2007) (Bruxelles, Peter Lang, 2010) e Tra prosa e poesia. Modernità di Sandro Penna (Roma, Edilet, 2010); nel 2010 ha curato per le edizioni Nerosubianco di Cuneo la raccolta di racconti postcoloniali Roma d'Abissinia. Asmara, Mogadiscio, Addis Abeba: cronache dai resti dell'impero. Ha curato il volume Il discorso sulla nazione nella letteratura italiana (Firenze, Cesati, 2012) e ha pubblicato la raccolta di racconti Di eredi non vedo traccia. Storie di tani, mericani e tripolini (Cuneo, Nerosubianco, 2012).

Université Libre de Bruxelles

Faculté de Philosophie et Lettres

Département de langues et littératures

Avenue F.D. Roosevelt 50

B-1050 Bruxelles (Belgio)

dcomberi@ulb.ac.be

\section{SUMMARY}

\section{Rewriting History}

Modalities of Representation of Italian Colonialism in Albania

Departing from the contemporary work of migrant authors of Albanian origin - Ornela Vorpsi, Anilda Ibrahimi and Elvira Dones - as well as from the theatrical monologue Italianesi by Saverio La Ruina, this article delves into the question of identity within 
postcolonial discourse. What role, in fact, do the Italian colonizers that remained behind in the liberated colonies play? How does their 'hybrid' identity help us to reflect upon colonial violence and upon the difficulties the colonizers themselves would have experienced internally when faced with accepting this violence? Additionally, the specific discussion of Albania, vis-à-vis narratives of contemporary migrants, allows us to reflect upon the Italian situation in particular, which, from unification to the present day offers specific material for the discussion of the relationship to, and the confronting of, the theme of 'otherness'. A broad definition of 'European' colonialism - that goes beyond the alleged African otherness and runs along the same lines as a redefinition vehemently proposed by new groups of migrants in Italy (in particular by those from the Albanian immigration wave of 1991) - allows one to question the Italian ambitions in regards to the Balkans and the Eastern expansion across national borders. 